Published in "Physical Review A - Atomic, Molecular and Optical Physics 86(5): 053427, 2012"

which should be cited to refer to this work.

\title{
Ground-state Hanle effect based on atomic alignment
}

\author{
Evelina Breschi* and Antoine Weis \\ Physics Department, University of Fribourg, CH-1700 Fribourg, Switzerland
}

\begin{abstract}
We have studied the ground-state Hanle effect (GSHE) excited by linearly polarized laser light on the $D_{1}$ line of cesium atoms. We have solved algebraically the Liouville equation using the irreducible tensor formalism, and derive an analytical expression for the resonance line shapes in a magnetic field of arbitrary direction. The model predictions are in excellent agreement with experimental observations in various field geometries. Our model is valid for arbitrary $F \rightarrow F^{\prime}$ transitions in the low-power limit. We discuss the relation between the GSHE and electromagnetically induced transparency or absorption. Our approach allows a full understanding of the mechanism of the GSHE and provides tools for quantifying the resonance contrast, a crucial parameter for metrological applications of level crossing resonances.
\end{abstract}

\section{INTRODUCTION}

The macroscopic spin polarization produced by optical pumping in an ensemble of paramagnetic atoms changes the optical absorption and dispersion characteristics of the medium. This property forms the basis for the optical detection of perturbations that alter the medium's (steady-state or dynamically evolving) magnetization that is proportional to its spin polarization. The principle of optical creation and detection of spin polarization is widely applied in atomic magnetometers [1], atomic clocks [2], atom interferometry [3], detection of slow light [4], optical information storage [5], or the search for violations of discrete symmetries in atoms and molecules [6], to name just a few.

The simplest of all polarization-perturbing interactions that can be detected by optical means is the effect of a suitably oriented external dc magnetic field on the atomic sample. In practice, one records the power $P$ of the light beam traversing the atomic medium as a function of the magnitude $B$ of a magnetic field that is scanned from negative to positive values. The depolarizing effect of the magnetic field then manifests itself as a resonance, centered at $B=0$; this effect is called the ground-state Hanle effect (GSHE). The width of the resonance is proportional to the relaxation rate of the spin coherence produced by optical pumping.

Hanle had first observed the effect named after him in 1924 as a magnetic field induced depolarization of resonance fluorescence [7]. The origin of this linear Hanle effect (LHE) is the Larmor precession and relaxation of spin polarization in the excited state of the atoms. Since the excited state spin coherence relaxes on time scales in the ns range, while ground-state spin polarization may live as long as seconds, the linewidths of the ground-state Hanle resonances are many orders of magnitude narrower than the corresponding linear Hanle resonance, hence their use in precision high resolution applications.

The observation of the GSHE using circularly polarized resonance light was first reported by Lehmann and CohenTannoudji in 1964 [8]. Recently, a detailed study of the characteristics of the GSHE was used to determine the

\footnotetext{
*evelina.breschi@unifr.ch
}

longitudinal and transverse relaxation times of spin-oriented atoms [9]. The GSHE with linearly polarized light was reported in the late sixties and a review of the early work can be found in Ref. [6]. In recent years it has attracted a renewed interest in the context of coherent population trapping (CPT) spectroscopy [10-12].

In the present paper we use a simple formalism to derive an algebraic expression for the line shape of ground-state Hanle resonances observed with linearly polarized light in arbitrarily oriented magnetic fields. The model predictions are compared to experimental spectra recorded in various field geometries.

\section{A. Scope of the present work}

Our study is complementary to related work described in the literature. Some authors model the complex spin structures occurring in alkalis in terms of three- or four-level systems $[13,14]$, yielding analytical results that provide a qualitative description of the main resonance features. Another approach uses time consuming numerical calculations that take the complete spin structure and arbitrary light intensities into account $[15,16]$. However, the latter approach is not well suited to extract characteristic system parameters, such as relaxation rate(s), by data fitting procedures. A third approach just uses empirical line shape functions to analyze the resonances [17], but gives little insight into the underlying mechanisms of the GSHE.

We have studied the GSHE excited with linearly polarized laser light in geometries illustrated in Fig. 1(a). A linearly polarized resonant laser beam propagates through Cs vapor in an evacuated cell. We record the variation of the transmitted power $P(B)$ when the magnitude of a magnetic field $\vec{B}$, oriented at an angle $\theta$ with respect to the light polarization, is scanned around $B=0$. The symmetry of the laser beam interacting with the atoms is determined by the orientation of the light polarization, defining the $z$ axis. As a consequence, all possible field geometries are fully described by a single parameter, viz., the angle $\theta$ between the laser polarization and the magnetic field.

The paper is organized as follows: In Sec. II we describe in detail our theoretical approach for modeling the shape of the resonances. Section III addresses details of the experimental apparatus and the methods deployed, and in Secs. IV to VI 


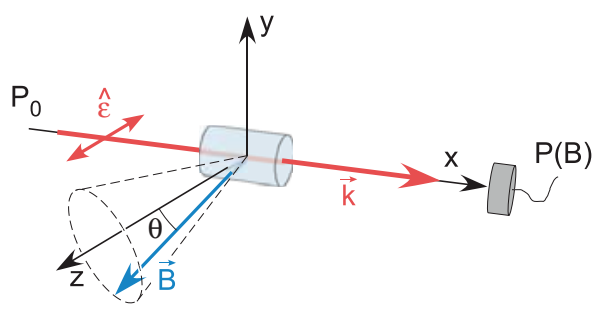

FIG. 1. (Color online) General geometry for observing GSHE with linearly polarized light and an arbitrarily oriented magnetic field.

we compare experimental results obtained with different magnetic field configurations to the model predictions. We conclude by discussing in Sec. VII the connection between the observations and interpretations put forward here with alternative interpretations of the GSHE.

\section{THEORETICAL MODEL}

We derive general algebraic expressions that describe the line shape of ground-state Hanle resonances with linearly polarized light in arbitrary magnetic field geometries. The derived equations are valid in the low light power limit (defined below), and, more importantly, they apply for $F \rightarrow$ $F^{\prime}$ transitions between states with arbitrary angular momenta $F$ and $F^{\prime}$.

We follow a three-step approach [6] that has been applied successfully in the past to model the magneto-optical resonances observed with the nonlinear Faraday effect [18,19], with magnetometers based on atomic alignment [20], and in the GSHE with circularly polarized light [9]. The three steps consist in considering (1) the preparation of spin polarization by optical pumping, (2) the evolution of the polarization in the magnetic field, and finally (3) the detection of the evolved polarization.

(1) Alignment creation (step 1). As a first step we consider the creation of spin alignment by optical pumping with linearly polarized light tuned to an $F \rightarrow F^{\prime}$ transition. It is known [21] that light driving an electric dipole transition couples only the spin multipole moments $m_{0,0}$ (total level population), $m_{1, q}$ (spin orientation vector, also known as Bloch vector), and $m_{2, q}$ (the five components of the spin alignment tensor) of the atomic ground state. Optical pumping with linearly polarized light creates only $k$-even longitudinal $(q=0)$ multipole moments $m_{k=\text { even, } 0}$ [22,23], when the quantization axis $z$ is chosen along the light polarization. As a consequence, the medium absorption is fully characterized by $m_{0,0}$ (which may be affected by hyperfine pumping) and the longitudinal alignment component $m_{2,0}$. With the choice of quantization axis along $\widehat{\varepsilon}, m_{2,0}$ is proportional to the expectation value $m_{2,0} \propto\left\langle\vec{F}^{2}-3 F_{z}^{2}\right\rangle$ where the proportionality constant is not relevant in the present context. Below, we will call $m_{2,0}^{\mathrm{eq}}$ the equilibrium longitudinal alignment created in step (1).

(2) Alignment evolution (step 2). In a second step we consider the evolution of the alignment produced in step (1) under the combined action of Larmor precession and relaxation. Since the laser frequency is actively kept resonant with the hyperfine transition under investigation, and since we consider only the low-power limit, we can neglect multipole mixing effects, such as orientation-alignment conversion induced by the optical field [24].

We start from the Liouville equation describing the dynamics of the density matrix $\rho$ of the ground state

$$
\hbar i \frac{d \rho(t)}{d t}=[H(t), \rho(t)]+\Gamma\left[\rho(t)-\rho^{\mathrm{eq}}\right],
$$

where $H(t)=-\vec{\mu} \cdot \vec{B}$ is the Zeeman Hamiltonian, $\Gamma$ the relaxation matrix, and $\rho^{\text {eq }}$ the equilibrium value of $\rho(t)$ for $\vec{B}=0$, i.e., the value of $\rho$ prepared by optical pumping in the first step.

The density matrix can be decomposed into irreducible spherical multipole moments [22] according to

$$
\rho=\sum_{k=0}^{2 F} \sum_{q=-k}^{k} m_{k, q} T_{q}^{(k)}(F),
$$

where $T_{q}^{(k)}$ are irreducible tensor operators [20] acting in the spin space $(F)$ of the ground state, and $m_{k, q}$ the atomic multipoles moments that describe the medium's spin polarization. Inserting (2) into Eq. (1), and making use of the commutator properties of the $T_{q}^{(k)}$ with the angular momentum operators $F_{q}$ yields a set of five equivalent algebraic equations of motion for the multipole moments $m_{2, q}$, that can be expressed as

$$
\begin{aligned}
\frac{d m_{2, q}}{d t}= & \sum_{q^{\prime}=-2}^{2}\left(\mathcal{O}_{q, q^{\prime}}^{(2)}-\delta_{q, q^{\prime}} \Gamma_{\left|q^{\prime}\right|}\right) m_{2, q^{\prime}} \\
& +\sum_{q^{\prime}=-2}^{2} \delta_{0, q^{\prime}} \Gamma_{\left|q^{\prime}\right|} m_{2, q^{\prime}}^{\mathrm{eq}},
\end{aligned}
$$

where the $\delta_{i, j}$ are Kronecker symbols, and where the evolution matrix is given by

$\mathcal{O}_{q, q^{\prime}}^{(2)}=\left(\begin{array}{ccccc}-2 i \omega_{z} & i \omega_{-} & 0 & 0 & 0 \\ -i \omega_{+} & -i \omega_{z} & \sqrt{\frac{3}{2}} i \omega_{-} & 0 & 0 \\ 0 & -\sqrt{\frac{3}{2}} i \omega_{+} & 0 & \sqrt{\frac{3}{2}} i \omega_{-} & 0 \\ 0 & 0 & -\sqrt{\frac{3}{2}} i \omega_{+} & i \omega_{z} & i \omega_{-} \\ 0 & 0 & 0 & -i \omega_{+} & 2 i \omega_{z}\end{array}\right)$

in which $\omega_{ \pm}=\omega_{x} \pm i \omega_{y}$ with $\omega_{i}=\gamma_{F} B_{i}$ are the Larmor frequencies associated with each Cartesian component of the magnetic field $\vec{B}, \gamma_{F}$ being the gyromagnetic factor. The second term in Eq. (4) describes the relaxation at rates $\Gamma_{|q|}$ of the alignment components $m_{2, q}$. The last term in Eq. (4) is a source term that describes the creation of the longitudinal alignment $m_{2,0}^{\mathrm{eq}}$ in step (1). It ensures that in the absence of a magnetic field $(\vec{\omega}=0)$, the equilibrium alignment is given by $\left(0,0, m_{2,0}^{\mathrm{eq}}, 0,0\right)$.

The steady-state solutions of Eq. (5) yield the steady-state values of the transverse alignment components (coherences) $m_{2, q \neq 0}$, as well as the longitudinal alignment $m_{2,0}$, which is the only relevant quantity for the next step.

(3) Alignment detection (step 3). The final step consists in expressing the power transmitted by the medium in terms of the medium's steady-state alignment. For this we consider 
the Lambert-Beer law, which states that the power $P$ of a near-resonant laser beam transmitted by an atomic vapor obeys

$$
P=P_{0} e^{-\kappa_{0}\left(m_{2,0}\right) \mathcal{D}(\delta \omega) L},
$$

where $P_{0}$ is the light power entering the cell, $L$ the thickness of the atomic vapor, $\mathcal{D}(\delta \omega)$ the Doppler line shape function with $\mathcal{D}(0)=1$, and $\delta \omega$ the laser detuning, i.e., the difference between the laser frequency, and the atomic resonance frequency of the hyperfine transition. $\kappa_{0}\left(m_{2,0}\right)$ is the peak absorption coefficient that depends on the degree of alignment of the medium. One can show that is related to the absorption coefficient $\kappa_{0}^{\text {unpol }}$ of the unpolarized medium by

$$
\kappa_{0}\left(m_{2,0}\right)=\kappa_{0}^{\text {unpol }}\left(1-\alpha_{F, F^{\prime}} m_{2,0}\right),
$$

where the specific alignment analyzing power $\alpha_{F, F^{\prime}}$ of the $F \rightarrow F^{\prime}$ transition is considered to be a constant with an irrelevant numerical value for the present study.

\section{The ground-state Hanle resonances}

The steady-state solutions $d m_{k, q} / d t=0$ of Eq. (5) yield for the relevant alignment component $m_{20}$

$$
\frac{m_{20}}{m_{2,0}^{\mathrm{eq}}}=\frac{\Gamma_{0}\left[\omega_{\|}^{2}\left(4 \Gamma_{1}^{2}+\Gamma_{2}^{2}-4 \omega_{\perp}^{2}\right)+4 \omega_{\|}^{4}+\left(\Gamma_{1} \Gamma_{2}+\omega_{\perp}^{2}\right)^{2}\right]}{3 \omega_{\perp}^{2}\left[4 \Gamma_{1} \omega_{\|}^{2}+\Gamma_{2}\left(\Gamma_{1} \Gamma_{2}+\omega_{\perp}^{2}\right)\right]+\Gamma_{0}\left[4 \omega_{\|}^{4}+\omega_{\|}^{2}\left(4 \Gamma_{1}^{2}+\Gamma_{2}^{2}-4 \omega_{\perp}^{2}\right)+\left(\Gamma_{1} \Gamma_{2}+\omega_{\perp}^{2}\right)^{2}\right]},
$$

where we have introduced the longitudinal and transverse Larmor frequencies $\omega_{\|}=\omega_{z}$ and $\omega_{\perp}=\left|\omega_{ \pm}\right|=\sqrt{\omega_{x}^{2}+\omega_{y}^{2}}$, respectively.

Under the assumption of equal relaxation rates $\Gamma_{0}=\Gamma_{1}=$ $\Gamma_{2} \equiv \Gamma$, Eq. (8) can be simplified considerably, yielding

$$
\frac{m_{2,0}}{m_{2,0}^{\mathrm{eq}}}=1-\frac{3 \omega_{\perp}^{2}\left(\Gamma^{2}+\omega_{\perp}^{2}+4 \omega_{\|}^{2}\right)}{\left(\Gamma^{2}+\omega_{\perp}^{2}+\omega_{\|}^{2}\right)\left(\Gamma^{2}+4 \omega_{\perp}^{2}+4 \omega_{\|}^{2}\right)} .
$$

Introducing further the dimensionless longitudinal and transverse Cartesian field variables $\beta_{\|} \equiv \omega_{\|} / \Gamma$ and $\beta_{\perp} \equiv$ $\omega_{\perp} / \Gamma$, respectively, Eq. (9) can be expressed, after rearranging the terms, as

$$
\mu_{2,0}\left(\beta_{\|}, \beta_{\perp}\right)=\frac{1}{4}+\frac{3}{4} \frac{16 \beta_{\|}^{4}+8 \beta_{\|}^{2}+1}{4 \beta_{\|}^{2}+4 \beta_{\perp}^{2}+1}-3 \frac{\beta_{\|}^{4}+\beta_{\|}^{2}}{\beta_{\|}^{2}+\beta_{\perp}^{2}+1},
$$

where we have defined $\mu_{2,0}\left(\beta_{\|}, \beta_{\perp}\right) \equiv m_{2,0}\left(\beta_{\|}, \beta_{\perp}\right) / m_{2,0}^{\text {eq }}$, which measures the detected alignment in units of the maximal (equilibrium) alignment obtained by optical pumping. From Eq. (10) one sees that the general resonance line shape consists of two resonances with different widths and of opposite sign that are superposed on a field independent background. In Secs. IV to VI below we will analyze the structure of Eq. (10) in more detail for specific experimental geometries.

\section{EXPERIMENTAL PROCEDURES}

\section{A. Experimental apparatus}

We have studied the GSHE in Cs vapor using radiation from a laser beam whose frequency was actively stabilized to the $F=4 \rightarrow 3$ transition of the $D_{1}$ line, which is the most efficient for producing (and probing) atomic alignment.

The atomic vapor is contained in an evacuated uncoated (20 mm long, $20 \mathrm{~mm}$ diameter) Pyrex cell kept at room temperature. This choice of cell was dictated by our wish to ensure the equality of the three relaxation rates $\Gamma_{0,1,2}$ of the atomic alignment, since a single atom-wall collision in a vacuum cell completely randomizes all atomic spin polarization components. The price we pay with this choice is the fact that relaxation is no longer exponential, since it is determined by the distribution of the atom-field interaction times that occur between steps (1) and (3) in the three-step model. The ensuing line shapes are given by the Fourier transform of the interaction time distribution, and depend on the transverse laser intensity profile and the radial atomic velocity distribution [25]. As a consequence, the resonances do not have Lorentzian shapes. This effect leads to a minor correction that is not particularly relevant in our context; however, for the sake of completeness we will address this effect in Appendix B.

The cell is mounted in a 992-mm-long double-layer $\mu$-metal shield with an inner diameter of $209 \mathrm{~mm}$. The laser light is carried by a monomode polarization maintaining fiber. The output beam is collimated and expanded to about $10 \mathrm{~mm}$ by a telescope. The central part of the expanded beam is passed through a diaphragm producing a 6 -mm-diameter beam. A $\lambda / 2$ plate and a linear polarizer are used to control the power $P_{0}$ of the beam and its polarization orientation. The light transmitted through the cell is detected by a biased photodiode followed by a current-voltage converter.

A magnetic field in an arbitrary direction can be applied to the cell by means of two pairs of rectangular coils mounted in Helmholtz configuration and a solenoid. In Appendix A we discuss the magnetic field calibration procedures. GSHE spectra are recorded by measuring the dependence of the transmitted light power $P$ on the magnitude of the magnetic field. The current through the (Helmholtz or solenoid) coil producing the scanned field is ramped symmetrically from negative to positive values, and the photodiode signal $P(B)$, as well as the current-controlling voltage ramp, is recorded (and averaged) on a digital storage oscilloscope for offline analysis.

\section{B. Choosing the optimum laser power}

Optical pumping with linearly polarized light produces subsequently all even multipole moments $m_{2,0}, m_{4,0}, \ldots$, up to $m_{2 F, 0}\left(=m_{8,0}\right.$, for the $4 \rightarrow 3$ transition $)$, when the incident 


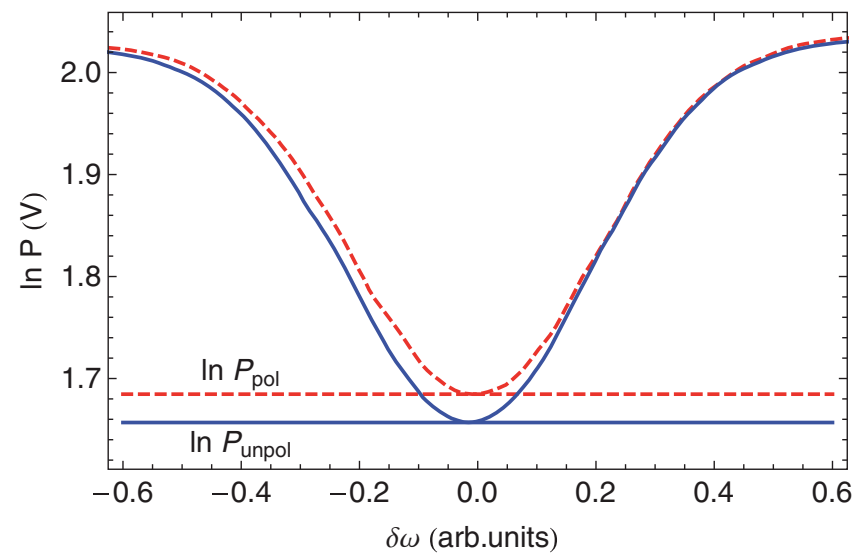

FIG. 2. (Color online) Linearized transmission spectra of the $4 \rightarrow$ 3 transition with polarized (dashed line) and unpolarized (solid line) atoms $\left(P_{0} \approx 10 \mu \mathrm{W}\right)$. $\ln P_{\text {unpol }}$ and $\ln P_{\mathrm{pol}}$ are the on-resonance signals from unpolarized and polarized atoms, respectively.

laser power $P_{0}$ is gradually increased. We recall that we chose the quantization axis for the $m_{(k, q)}$ representation along the light polarization.

The alignment $m_{2,0}$ is produced first, and we assume that the medium has no higher multipole moments as long as the dependence of $m_{2,0}$ on $P_{0}$ is linear. Since our model calculations are based on alignment contributions only, we have thus to make sure that the experiments are performed under conditions for which $m_{k>2,0} \approx 0$.

We determine the optimal power by using the fact thataccording to Eq. (10)—a strong longitudinal (i.e., parallel to the laser polarization vector) magnetic field $\beta_{\|} \gg \beta_{\perp}$ stabilizes the alignment to the value $m_{2,0}=m_{2,0}^{\text {eq }}$ produced by optical pumping. On the other hand, a strong transverse field $\beta_{\perp} \gg \beta_{\|}$ will completely destroy the alignment $\left(m_{2,0}^{\text {eq }}=0\right)$. We record transmission spectra $P(\delta \omega)$ by scanning the laser frequency over the $4 \rightarrow 3$ transition under alignment-stabilizing $\left(\beta_{\|}=\right.$ $10)$ and alignment-destroying $\left(\beta_{\perp}=10\right)$ conditions. A typical example of such spectra is shown in Fig. 2, where we have linearized the transmission signals by taking the (natural) logarithm of the photodiode signals. On the $4 \rightarrow 3$ transition studied here, the absorption coefficient in the aligned vapor is reduced compared to the unpolarized vapor, thus exhibiting electromagnetically induced transparency (EIT).

According to Eqs. (6) and (7), $\ln P$ is given by

$$
\ln P(\delta \omega)=\ln P_{0}-\kappa_{0} L \mathcal{D}(\delta \omega),
$$

where $\delta \omega$ is the laser detuning from the $4 \rightarrow 3$ transition. For each spectrum, we fit the function (11) to the data, the on-resonance $(\delta \omega=0)$ optical density $\kappa_{0} L$ being one of the fit parameters. ${ }^{1}$ With a transverse magnetic field applied (unpolarized medium), the optical density is given by $\left(\kappa_{0} L\right)_{\perp}=\kappa_{0}^{\text {unpol }} L$, while in presence of a polarization stabilizing longitudinal field, one has

\footnotetext{
${ }^{1}$ In the context of the present paper we refer to $\kappa_{0} L$ as the optical density. It is related to the standard definition of the optical density OD used in optics via $10^{-\mathrm{OD}} \equiv e^{-\kappa_{0} L}$.
}

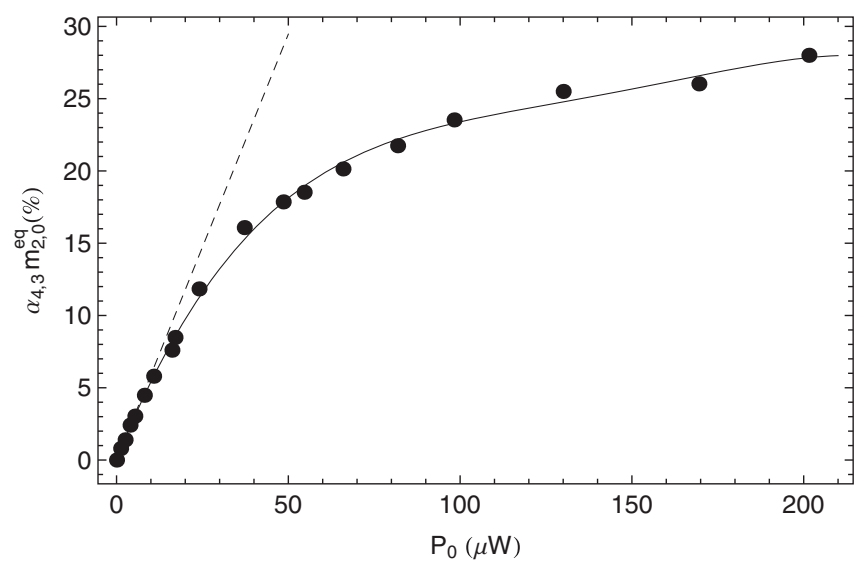

FIG. 3. Atomic alignment as a function of the laser power: the dots are the experimental points; the solid line is a polynomial fit, while the dashed line is the linear part of the polynomial fit.

$$
\begin{array}{r}
\left(\kappa_{0} L\right)_{\|}=\left(\kappa_{0}^{\mathrm{unpol}} L\right)\left(1-\alpha_{4,3} m_{2,0}^{\mathrm{eq}}\right) . \text { The quantity } \\
\frac{\left(\kappa_{0} L\right)_{\perp}-\left(\kappa_{0} L\right)_{\|}}{\left(\kappa_{0} L\right)_{\perp}} \equiv \alpha_{4,3} m_{2,0}^{\mathrm{eq}}
\end{array}
$$

thus represents a measure that is proportional to the atomic alignment produced by optical pumping. In Fig. 2, the horizontal dashed lines indicate the (on-resonance) signals $\ln P_{\text {unpol }}$ (solid) and $\ln P_{\text {pol }}$ (dashed) produced by unpolarized and polarized atoms, respectively.

Figure 3 shows how $\alpha_{4,3} m_{2,0}^{\mathrm{eq}}$ extracted from the experimental data using (12) depends on the incident laser power $P_{0}$. The solid line is a polynomial function, which allows us to infer the region in which $m_{2,0}^{\text {eq }}$ grows linearly with $P_{0}$. Based on this result we have chosen to carry out the experiments with a laser power of $\approx 10 \mu \mathrm{W}$, which corresponds to an intensity of $\approx 35 \mu \mathrm{W} / \mathrm{cm}^{2}$.

We note that a corresponding method has been described in Ref. [9] to infer the optimal power in atomic orientation-based GSHE experiments.

\section{Extracting $\mu_{2,0}$ from the transmission spectra}

The ground-state Hanle resonances are recorded by measuring the magnetic field dependence $P(\beta)$ of the transmitted laser power, when the laser frequency is actively stabilized to the center of the $4 \rightarrow 3$ transition $(\delta \omega=0)$. Following the previous discussion, one sees that $P(\beta)$ is bound to lie in the range $\left[P_{\mathrm{unpol}}, P_{\mathrm{pol}}\right]$. This range is determined by the range of possible alignments $0 \leqslant m_{2,0} \leqslant m_{2,0}^{\text {eq }}$, which is equivalent to $0 \leqslant \mu_{2,0} \leqslant 1$.

A straightforward consequence is that one can infer from the simple measurement in Fig. 2 the fundamental limit of the signal-to-(shot)noise ratio for a given experimental configuration. This result is important in view of applications of the ground-state Hanle resonances as reference signals in quantum sensors. The method is not restricted to the GSHE, but can be applied to a larger set of related resonance effects in spin polarized atoms. 
The magnetic field dependent relative alignment $\mu_{2,0}(\beta)$ can be extracted from the measured $P(\beta)$ dependence via

$$
\mu_{2,0}(\beta)=\frac{\ln P(\beta)-\ln P_{\text {unpol }}}{\ln P_{\mathrm{pol}}-\ln P_{\text {unpol }}},
$$

where $P_{\mathrm{pol}}$ and $P_{\text {unpol }}$ are the values determined in the auxiliary absorption measurement described in Sec. III B.

We note that the use of the relative alignment $\mu_{2,0}$, both in the theoretical model and in the analysis of the experimental spectra, does not require the knowledge of the alignment analyzing power $\alpha_{F, F^{\prime}}$.

In the following three sections we will describe GSHE experiments performed in different field geometries and compare the results to the model predictions derived in Sec. II.

\section{ORIENTATIONAL DEPENDENCE OF THE GSHE}

We will first address the dependence of the Hanle resonance line shapes on the angle $\theta$ between the magnetic field orientation $\hat{B}$ and the light polarization vector $\hat{\varepsilon}$. The theoretical line shapes are obtained by rewriting (10) in terms of the spherical field variables $\beta$ and $\theta$, where $\beta_{\|} \equiv \beta \cos \theta, \beta_{\perp} \equiv \beta \sin \theta$, and $\beta^{2} \equiv \beta_{\|}^{2}+\beta_{\perp}^{2}$, as

$$
\mu_{2,0}(\beta, \theta)=\left(\frac{3 \cos ^{2} \theta-1}{2}\right)^{2}+\frac{3}{4} \frac{\sin ^{2}(2 \theta)}{1+\beta^{2}}+\frac{3}{4} \frac{\sin ^{4} \theta}{1+4 \beta^{2}},
$$

which can be put in the very compact form

$$
\mu_{2,0}(\beta, \theta)=\sum_{q=-2}^{2} \frac{\left|C_{2, q}(\theta, 0)\right|^{2}}{1+q^{2} \beta^{2}},
$$

where the $C_{2, q}$ are the components of the rank 2 unit tensor which are related to the spherical harmonics $Y_{2, q}$ by $C_{2, q}(\theta, \varphi)=\sqrt{4 \pi / 5} Y_{2, q}(\theta, \varphi)$.

It is interesting to note that the GSHE line shapes induced by circularly polarized laser light are fully described [9] by the corresponding expression

$$
\mu_{1,0}(\beta, \theta)=\sum_{q=-1}^{1} \frac{\left|C_{1, q}(\theta, 0)\right|^{2}}{1+q^{2} \beta^{2}}
$$

for the normalized vector polarization $\mu_{1,0}=m_{1,0} / m_{1,0}^{\mathrm{eq}}$, in which case $\theta$ refers to the angle between the scanned field and the laser propagation direction $\hat{k}$.

Equation (14) reflects again that the ground-state Hanle resonances with linearly polarized light are fully characterized by a single orientational parameter, viz., the angle $\theta$ between the magnetic field $\vec{B}$ and the light polarization vector $\hat{\varepsilon}$.

Figure 4 represents a series of ground-state Hanle resonances recorded by scanning $B_{y}$ (Fig. 1) for various orientations of $\hat{\varepsilon}$. Since the signals depend only on the relative orientation of $\hat{B}$ and $\hat{\varepsilon}$, we have opted to vary the direction of $\hat{\varepsilon}$ (by means of a $\lambda / 2$ plate) rather than changing the (experimentally more demanding) direction of $\hat{B}$.

The relative alignment $\mu_{2,0}$ was determined from the experimental spectra by applying (13). Together with the magnetic field abscissa given in terms of the dimensionless magnetic field parameter $\beta=\beta_{y}$, each Hanle spectrum $\mu_{2,0}(\beta)$ can be fitted by the function (14) on an absolute scale, with $\theta$ being the only fit parameter. The fitted values of $\theta$ are given, together with their statistical uncertainties as inserts in each spectrum. The errors $\Delta \theta \approx 0.5^{\circ}$ are compatible with the experimental angular adjustment of $\theta$.

One sees that the line shapes are not perfectly described by the theoretical line shape (14). As mentioned earlier (and addressed in Appendix B), this is a consequence of the use of an uncoated cell without buffer gas (chosen to have $\Gamma_{0}=\Gamma_{1}=$ $\Gamma_{2}$ ), in which the relaxation is known to be nonexponential, and hence the line shapes to be non-Lorentzian [26]. We note nevertheless that the peak value (polarized level) and the far wing background are well reproduced by the fit function, which allows us to determine the signal amplitude $A$ and the signal background $b g$. According to Eq. (14), the $\theta$ dependence of the amplitude and background are given by

$$
A(\theta) \equiv \mu_{2,0}(0, \theta)-\mu_{2,0}(\infty, \theta)=1-\left(\frac{3 \cos ^{2} \theta-1}{2}\right)^{2}
$$

and

$$
\operatorname{bg}(\theta) \equiv \mu_{2,0}(\infty, \theta)=\left(\frac{3 \cos ^{2} \theta-1}{2}\right)^{2},
$$

respectively.

Figure 5 shows the angular dependencies of the complementary quantities $A(\theta)$ and $b g(\theta)$ inferred from the experimental data, together with the model functions (17) and (18). We note that the model functions are not fitted to the data here, but are defined on an absolute scale. A maximal contrast of $100 \%(A=1)$ is obtained at the magic angle $\theta^{*}=54.74^{\circ}$, which obeys $\cos ^{2} \theta^{*}=1 / 3$.

The dependence of the full width at half maximum (FWHM) linewidths $\Delta \beta$ on the field orientation $\theta$ can be inferred from Eq. (14) and is given by

$$
\Delta \beta=\sqrt{\frac{15 \cos ^{2} \theta-3+\sqrt{369 \cos ^{4} \theta+6 \cos ^{2} \theta+25}}{6 \cos ^{2} \theta+2}},
$$

a function that varies smoothly from $\Delta \beta=2$, i.e., $\Delta \omega=2 \Gamma$ at $\theta=0$, to $\Delta \beta=1$, i.e., $\Delta \omega=\Gamma$ at $\theta=\pi / 2$ (top curve of Fig. 6).

When optimizing the sensitivity of the GSHE for metrological applications, such as atomic magnetometry, the relevant figure-of-merit (under the assumption that the signal noise is field independent) is given by the ratio $F_{\text {om }} \equiv A / \Delta \beta$ of the resonance amplitude and the resonance linewidth. The bottom curve of Fig. 6 shows the expected angular dependence of the $F_{\text {om }}$, based on Eqs. (17) and (19). The curve illustrates that the best $F_{\text {om }}$ is obtained with a purely transverse $(\theta=\pi / 2)$ field, and not at the magic angle, as one can naively expect.

The spectra discussed above can only be obtained when there are no residual magnetic field components in the plane perpendicular to the direction of the scanned field. In the following two sections we will analyze how the resonance line shapes are affected by the presence of a small constant residual magnetic field $\left(\beta_{p} \lesssim 1\right)$. We will address two specific geometries, viz., transverse and longitudinal Hanle resonances 

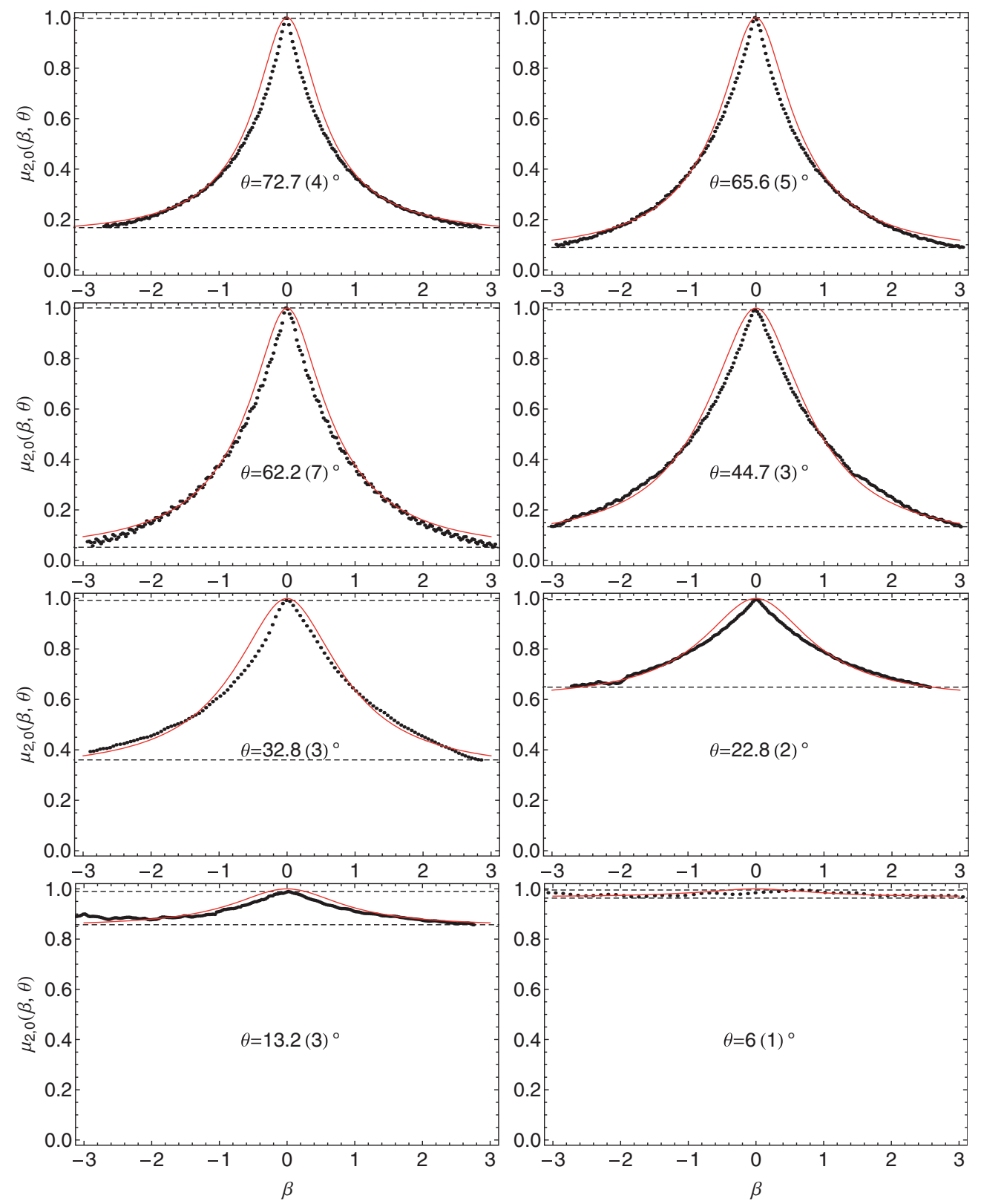

FIG. 4. (Color online) Line shape of Hanle resonances for different values of the magnetic field orientation. The values of $\theta$ represent the angle fit parameter with its error.

defined by the scanned field being, respectively, perpendicular or parallel to the light polarization.

\section{EFFECT OF RESIDUAL FIELDS ON THE TRANSVERSE GSHE}

When the amplitude of a magnetic field $\vec{\beta}_{\text {s }}$ that is perpendicular to $\hat{\varepsilon}$ (transverse geometry) is scanned in the absence of any residual field components, the line shape can be inferred from the Cartesian representation (10) to be

$$
\mu_{2,0}\left(\beta_{\|}=0, \beta_{\perp}=\beta_{\mathrm{s}}\right)=\frac{1}{4}+\frac{3}{4} \frac{1 / 4}{\beta_{\mathrm{s}}^{2}+1 / 4},
$$

which represents a Lorentzian with an amplitude of 3/4 and a FWHM width of 1 , that is superposed on a background of $1 / 4$.

In the experiments reported below we have applied a discrete set of small, constant parameter fields $\vec{\beta}_{\mathrm{p}}\left(\perp \vec{\beta}_{\mathrm{s}}\right)$ that simulate the presence of a residual field. Since the symmetry of the GSHE is given by the direction $\hat{\varepsilon}$, we have to consider two distinct orientations of the parameter fields, viz., $\hat{\beta}_{\mathrm{p}} \perp \hat{\varepsilon}$ (case 1 ) and $\hat{\beta}_{\mathrm{p}} \| \hat{\varepsilon}$ (case 2 ).

\section{A. Case 1: $\hat{\beta}_{\mathrm{p}} \perp \hat{\varepsilon}$}

In this case, both the scanned field $\vec{\beta}_{\mathrm{s}}$ and the parameter field $\vec{\beta}_{\mathrm{p}}$ are transverse fields, so that $\beta_{\perp}^{2}=\beta_{\mathrm{s}}^{2}+\beta_{\mathrm{p}}^{2}$, and the 


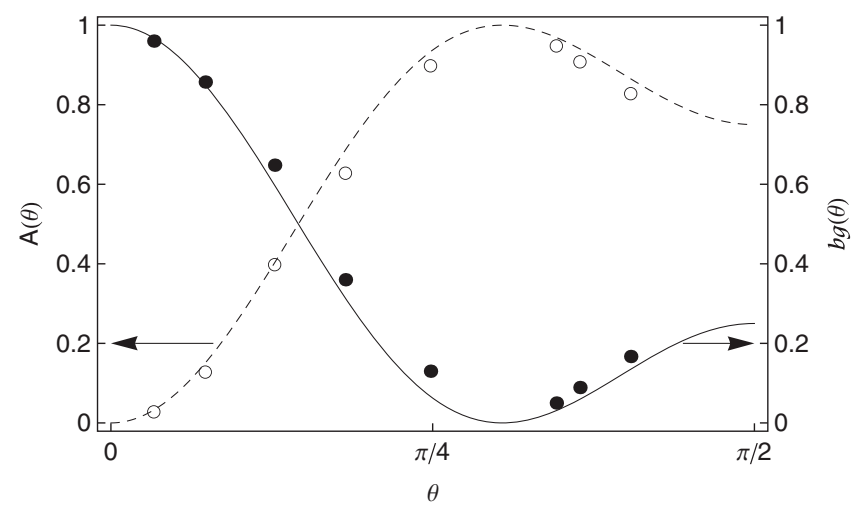

FIG. 5. Angular dependence of the resonance background, $b g(\theta)$ (solid dots), and contrast, $A(\theta)$ (open circles): dots and circles, experimental values; lines, Eqs. (18) and (17), respectively.

line shape (20) becomes

$$
\begin{aligned}
& \mu_{2,0}\left(\beta_{\|}=0, \beta_{\perp}=\left|\vec{\beta}_{\mathrm{s}}+\vec{\beta}_{\mathrm{p}}\right|\right) \\
& =\frac{1}{4}+\frac{3}{4} \frac{1 / 4}{\beta_{\mathrm{s}}^{2}+\beta_{\mathrm{p}}^{2}+1 / 4} \\
& =\frac{1}{4}+\frac{3}{4\left(\beta_{\mathrm{p}}^{2}+1 / 4\right)} \frac{\beta_{\mathrm{p}}^{2}+1 / 4}{\beta_{\mathrm{s}}^{2}+\left(\beta_{\mathrm{p}}^{2}+1 / 4\right)},
\end{aligned}
$$

which represents a family of upward pointing Lorentzians, whose amplitudes $A$ decrease with increasing residual field as

$$
A=\frac{3}{4} \frac{1 / 4}{\beta_{\mathrm{p}}^{2}+1 / 4},
$$

while their widths increase as

$$
\Delta \beta=\sqrt{1 / 4+\beta_{\mathrm{p}}^{2}} .
$$

The background of $1 / 4$ is not affected by the residual field.

We have recorded a family of such transverse Hanle resonances by scanning $\beta_{x}\left(\equiv \beta_{\mathrm{s}}\right)$, in the presence of a discrete set of transverse parameter fields $\beta_{y}$ ( $\equiv \beta_{\mathrm{p}}$ ) of different amplitudes. The results are shown in the left column of Fig. 7, together with the theoretical line shapes. The experimental data are well reproduced by the model.

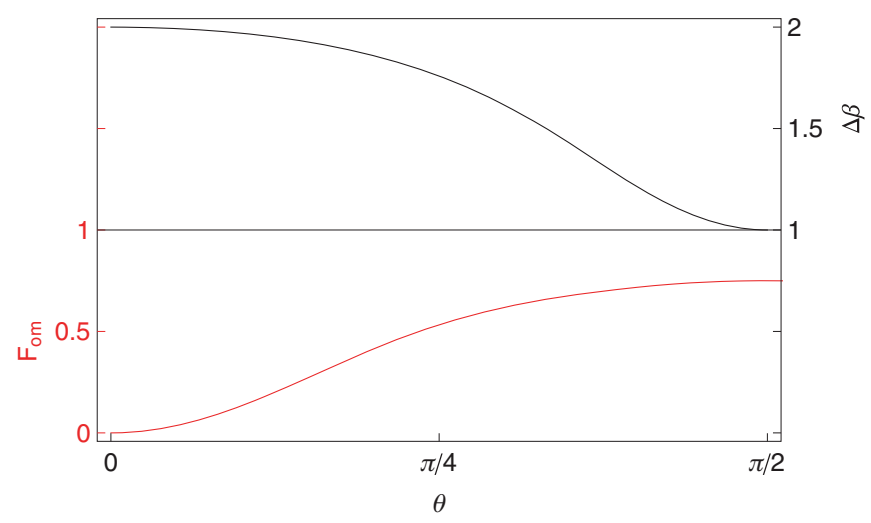

FIG. 6. (Color online) Angular dependence of the FWHM width and of the figure-of-merit $\left(F_{\mathrm{om}}\right)$ calculated using Eqs. (17) and (19).

\section{B. Case 2: $\hat{\beta}_{\mathrm{p}}=\hat{\boldsymbol{\varepsilon}}$}

Let us now examine how the transverse ground-state Hanle resonances are affected by a residual (parameter) field that is oriented along the light polarization. For this we rewrite (10) by identifying $\beta_{\perp}$ with $\beta_{\mathrm{s}}$ and $\beta_{\|}$with $\beta_{\mathrm{p}}$, yielding

$$
\begin{aligned}
\mu_{2,0}\left(\beta_{\mathrm{p}}, \beta_{\mathrm{s}}\right)= & \frac{1}{4}+\left(3 \beta_{\mathrm{p}}^{2}+3 / 4\right) \frac{\beta_{\mathrm{p}}^{2}+1 / 4}{\beta_{\mathrm{s}}^{2}+\left(\beta_{\mathrm{p}}^{2}+1 / 4\right)} \\
& -3 \beta_{\mathrm{p}}^{2} \frac{\beta_{\mathrm{p}}^{2}+1}{\beta_{\mathrm{s}}^{2}+\left(\beta_{\mathrm{p}}^{2}+1\right)} .
\end{aligned}
$$

The line shape consists of an upward pointing Lorentzian with a FWHM width of $2 \sqrt{\beta_{\mathrm{p}}^{2}+1 / 4}$ and of amplitude $3 \beta_{\mathrm{p}}^{2}+$ $3 / 4$, together with a broader, downward pointing Lorentzian of width $2 \sqrt{\beta_{\mathrm{p}}^{2}+1}$ and amplitude $3 \beta_{\mathrm{p}}^{2}$. For small parameter fields $\beta_{\mathrm{p}} \ll 1 / 2$, the narrower resonance dominates. At larger parameter fields, the broader negative resonance gives the line shape a pronounced $W$-shaped structure, while for very large residual fields $\left(\beta_{\mathrm{p}} \gg 1\right.$, not shown in the data), the line shape converges to a single upward Lorentzian of amplitude $3 / 4$ and width $2 \beta_{\mathrm{p}}$.

We have recorded a family of such transverse Hanle resonances by scanning $\beta_{x}\left(\equiv \beta_{\mathrm{s}}\right)$ in the presence of a discrete set of longitudinal parameter fields $\beta_{\mathrm{p}}\left(\equiv \beta_{z}\right.$ ) of different amplitudes. The results are shown in the central column of Fig. 7, together with the theoretical line shapes. The experimental data are well reproduced by the model.

\section{EFFECT OF RESIDUAL FIELDS ON THE LONGITUDINAL GSHE}

Last, we discuss the case when the scanned field is parallel to the light polarization $\hat{\varepsilon}$ (longitudinal geometry). In the absence of residual fields (orthogonal to the scanned field), the general formula predicts that there is no GSHE, i.e., $\mu_{2,0}\left(\beta_{\|}, \beta_{\perp}=0\right)=1$. However, any small residual field component will lead to the appearance of a resonance structure. In experiments we apply again a set of constant parameter fields $\vec{\beta}_{\mathrm{p}} \perp \vec{\varepsilon}$ to simulate the effect of residual fields. We apply the master expression (10) to the geometry of the longitudinal GSHE by identifying $\beta_{\|}$with $\beta_{\mathrm{s}}$ and $\beta_{\perp}$ with $\beta_{\mathrm{p}}$. Here we do not have to make a case distinction as for the transverse GSHE, since all transverse residual field orientations are equivalent.

Rewriting (10) to make the resonances relevant for the longitudinal GSHE appear explicitly yields

$$
\begin{aligned}
\mu_{2,0}\left(\beta_{\mathrm{s}}, \beta_{\mathrm{p}}\right)= & \frac{1}{4}+\frac{3 \beta_{\mathrm{p}}^{4}}{\beta_{\mathrm{p}}^{2}+1 / 4} \frac{\beta_{\mathrm{p}}^{2}+1 / 4}{\beta_{\mathrm{s}}^{2}+\left(\beta_{\mathrm{p}}^{2}+1 / 4\right)} \\
& -3 \beta_{\mathrm{p}}^{2} \frac{\beta_{\mathrm{p}}^{2}+1}{\beta_{\mathrm{s}}^{2}+\left(\beta_{\mathrm{p}}^{2}+1\right)} .
\end{aligned}
$$

As in case 2 of the transverse GSHE, the signal is composed of two resonances of opposite sign, whose amplitudes and linewidths can be identified easily from the parametrization used to write (26).

We have recorded a family of such longitudinal Hanle resonances by scanning $\beta_{z}\left(\equiv \beta_{\mathrm{s}}\right)$, in the presence of a discrete set of transverse residual fields $\beta_{\mathrm{p}}\left(\equiv \beta_{x}\right.$ ) of different amplitudes. 

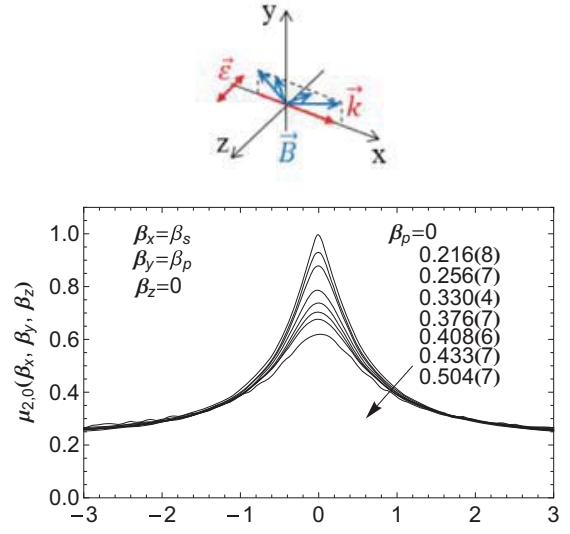

$\beta_{S}$

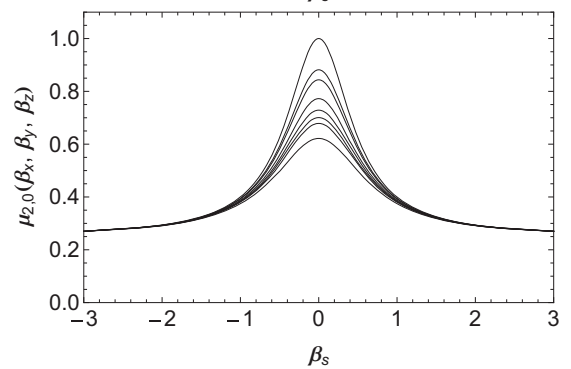

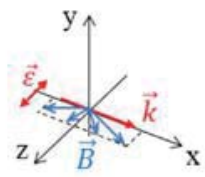

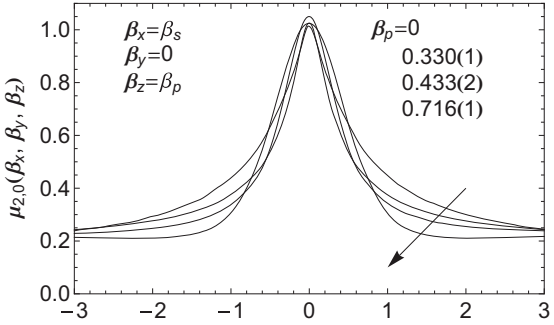

$\beta_{s}$

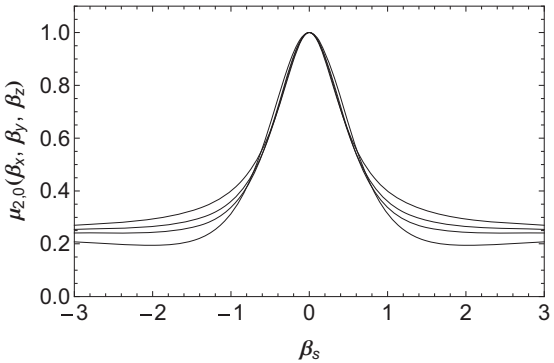

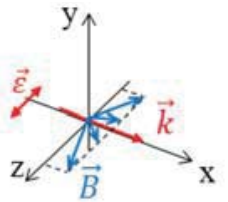

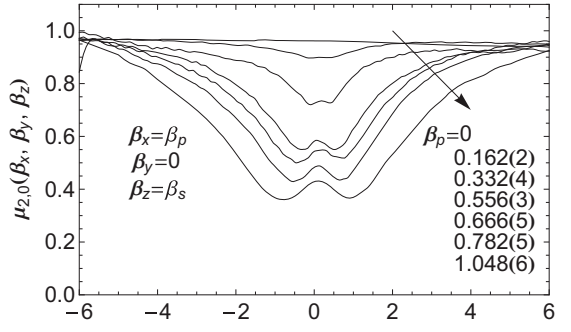

$\beta_{s}$

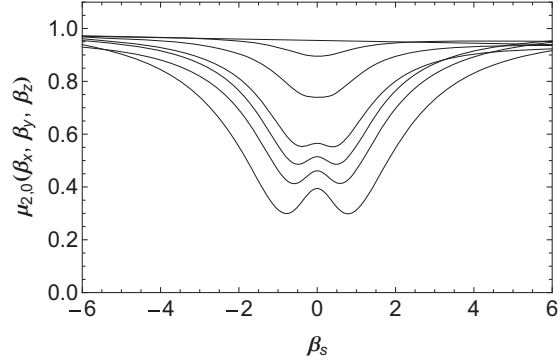

FIG. 7. (Color online) Comparison of experimental (center line) and theoretical (lower line) line shapes of the transverse and longitudinal Hanle resonances for a discrete set of parameter magnetic fields $\beta_{p}$ in the three relevant geometries summarized in the upper line. The parameter fields $\beta_{p}$ are given as insets, and the arrows point in the direction of increasing values of $\beta_{p}$.

The results are shown in the right column of Fig. 7, together with the theoretical line shapes. The experimental data are well reproduced by the model.

\section{DARK AND BRIGHT RESONANCES AND COHERENT POPULATION TRAPPING EFFECT}

In many cases, the redistribution of magnetic sublevel populations in the atomic ground state by optical pumping induces in the pumped medium an increased transparency for the pumping radiation. Our experiments were carried out on the $F=4 \rightarrow F^{\prime}=3$ transition, which indeed shows an increased transparency when the medium is polarized (Fig. 2 and left part of Fig. 8). One refers to this effect as an EIT. In the GSHE, the magnetic field destroys the spin polarization and hence the EIT effect produced by optical pumping. The transmitted power on the ground-state Hanle resonance (in the inset of Fig. 8) can vary between the extreme levels determined by the polarized and unpolarized medium. On resonance $(B=0)$ the transmission is maximal, i.e., the absorption minimal. This reduced absorption goes in pair with a reduced fluorescence, hence the name of dark resonance that is given to upward pointing Hanle resonances.

There is an important exception to the general rule that a pumped medium becomes transparent. As first pointed out by Kazantsev et al. [26], optical pumping on a closed transition between a ground state with angular momentum $F$ and an excited state with $F^{\prime}=F+1$ makes the polarized medium more absorbing for the pumping radiation than the unpolarized medium. In that case one speaks of electromagnetically induced absorption (EIA) [27,28]. The GSHE of closed (and to a lesser extend also of some open) $F \rightarrow F+1$ transitions therefore produces downward (bright) pointing resonances, in which the magnetic field destroys the electromagnetically induced absorption (Fig. 8, right).

The underlying physics of the GSHE lies in the magnetic field driven dynamic evolution of magnetic sublevel coherences. However, coherences have not been addressed explicitly by our theoretical model. By our proper choice of the quantization direction, only the longitudinal alignment-a quantity that is fully described by sublevel populationsintervenes in the preparation and detection processes. The magnetic field driven sublevel dynamics are treated implicitly

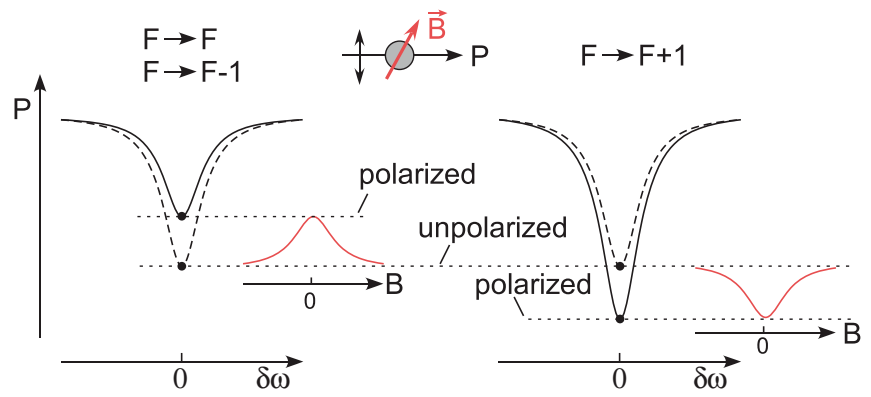

FIG. 8. (Color online) Effect of ground-state spin polarization on the transmission spectra of $F \rightarrow F, F-1$ transitions (left) and $F \rightarrow F+1$ transitions (right), showing EIT and EIA behavior, respectively. Dashed (solid) lines represent absorption lines for unpolarized (polarized) atoms. The magnetic field induced depolarization of the ground state leads to resonances of opposite signs in the two cases. 
by the equations of motion in step (2). The steady-state longitudinal alignment $m_{2,0}$ is derived as a solution of algebraic equations, in which the coherences are never used explicitly.

When choosing an alternative orientation of the quantization axis, as, e.g., along the magnetic field, the dark and bright states have to be described in terms of sublevel coherences. The magnetic field dynamics of step (2) in that case $(\hat{z}=\hat{B})$ are described by a diagonal Hamiltonian, and therefore straightforward to implement. However, the price that one pays for the trivial mathematics of step (2) is that the algebraic description of the state preparation and detection [steps (1) and (3)] becomes a nontrivial task, in particular when treating transitions with arbitrary $F$ and $F^{\prime}$.

In the literature the GSHE is sometimes referred to as a particular case of the coherent population trapping (CPT) effect $[29,30]$. From the discussion above, one could rather speak of a pure population trapping resonance when applying a treatment (like ours), where $\hat{z}=\hat{\varepsilon}$, and of a coherence trapping resonance in a treatment with the choice $\hat{z}=\hat{B}$.

\section{SUMMARY}

We have derived an algebraic expression for the line shape of ground-state Hanle resonances based on atomic alignment. The expression is valid for magnetic fields of arbitrary orientation and for transitions between states of arbitrary angular momenta. The approach is only valid for low laser intensities that create no atomic multipole moments higher than $m_{2,0}$. We have discussed in detail the case when all rank 2 multipole components, i.e., the longitudinal moment $m_{2,0}$ and the $\Delta m=1,2$ coherences $m_{2, \pm 1}$ and $m_{2, \pm 2}$, relax at the same rate. This case yields a particularly elegant mathematical expression (15) for the GSHE line shape in a field of arbitrary orientation.

Finally, we have shown that the amplitude, and hence the achievable contrast of the ground-state Hanle resonance, is determined and fundamentally limited by the degree of atomic alignment produced by optical pumping. This result is interesting in view of the applications of the GSHE and related effects in quantum sensors, whose performance is characterized in terms of the resonance contrast.

\section{ACKNOWLEDGMENTS}

This work is supported by SNF-Ambizione (Grant No. P Z00P2_131926) and by the Pool de Recherche of the University of Fribourg. We thank our colleagues from the FRAP group for the useful discussion, the mechanical workshop, and the electronics pool of the Physics Department for expert technical support.

\section{APPENDIX A: MAGNETIC FIELD CALIBRATION}

The magnetic field generating Helmholtz coils and the solenoid are driven by homemade voltage-controlled current sources. We have calibrated all coils using a procedure similar than the one described in Ref. [9]. We record magnetic resonance signals driven by a weak magnetic field that oscillates at a frequency $v_{\mathrm{rf}}$. When ramping the magnitude of a magnetic field from negative to positive values, the

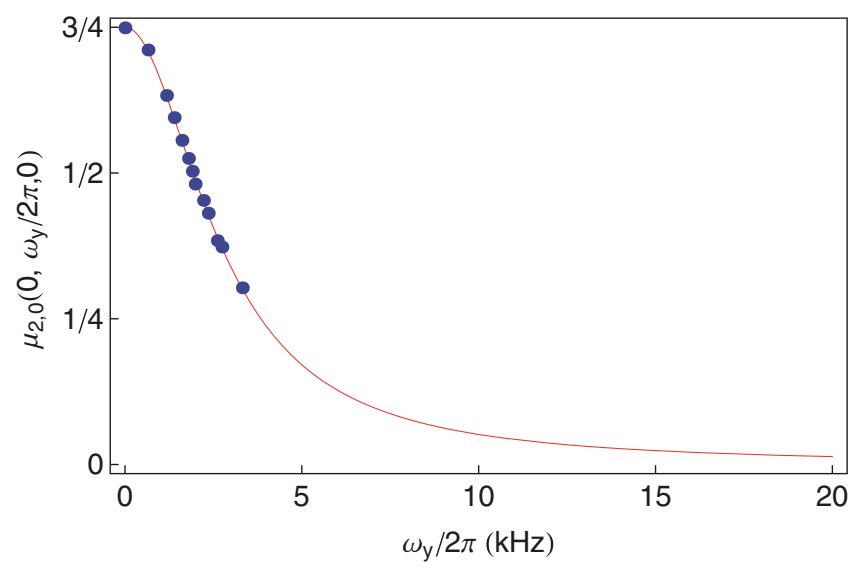

FIG. 9. (Color online) Variation of the amplitude of the transverse Hanle resonance in the presence of transverse fields $\omega_{y}$. A relaxation rate $\Gamma / 2 \pi=5.43(2) \mathrm{kHz}$ is inferred from a fit with the theoretical function (A1).

transmitted light power shows, in addition to the zero-field Hanle resonance, two magnetic resonance lines at field values given by $\omega_{L}= \pm \omega_{\mathrm{rf}}$, where $\omega_{L}=\gamma_{F} \sqrt{B_{x}^{2}+B_{y}^{2}+B_{z}^{2}}$ is the Larmor frequency corresponding to the modulus of the total magnetic field. By measuring how these resonant Larmor frequencies change with the current through each coil, the three coil calibration constants, measured in $\mathrm{Hz} / \mu \mathrm{A}$, can be inferred.

For characterizing the Larmor frequencies $\omega_{i}$ that correspond to the magnetic field components $B_{i}$ in terms of the dimensionless parameters $\beta_{i}=\omega_{i} / \Gamma$, we need furthermore a determination of the relaxation rate $\Gamma$. We infer $\Gamma$ from the study of Hanle resonances in a specific configuration using the following procedure. We record a set of transverse Hanle resonances using different parameter fields $\vec{\beta}_{\mathrm{p}} \perp \vec{\varepsilon}$ in the geometry of the case discussed in Sec. V A. When $\Gamma$ is not known, the amplitudes of these resonances read

$$
A\left(\omega_{\mathrm{p}}\right)=\frac{3}{4} \frac{\Gamma^{2}}{4 \omega_{\mathrm{p}}^{2}+\Gamma^{2}}
$$

in absolute units, where $\omega_{\mathrm{p}}$ is the Larmor frequency of the parameter field.

Figure 9 shows the dependence of the experimental amplitudes on $\omega_{\mathrm{p}}$, together with a fit using (A1). A relaxation rate of $\Gamma / 2 \pi=5.43(2) \mathrm{kHz}$ is found, that is used in all conversions of fields $B_{i}$ to the dimensionless units $\beta_{i}$. We note that we have used the amplitude dependence on $\beta_{\mathrm{p}}$ for the $\Gamma$ calibration, rather than the width dependence on $\beta_{\mathrm{p}}$, since the extracted amplitudes are more reliable than the widths, as discussed in Appendix B.

\section{APPENDIX B: TIME-OF-FLIGHT VS LORENTZIAN LINE SHAPES}

The Hanle resonance line shape is Lorentzian if homogeneous broadening mechanisms, such as pressure broadening in a buffer gas or effects from the finite laser linewidth [31], dominate the coherence relaxation rates. A review of coherent relaxation rates effects in the case of electromagnetically induced transparency was reported by Xiao [32]. 

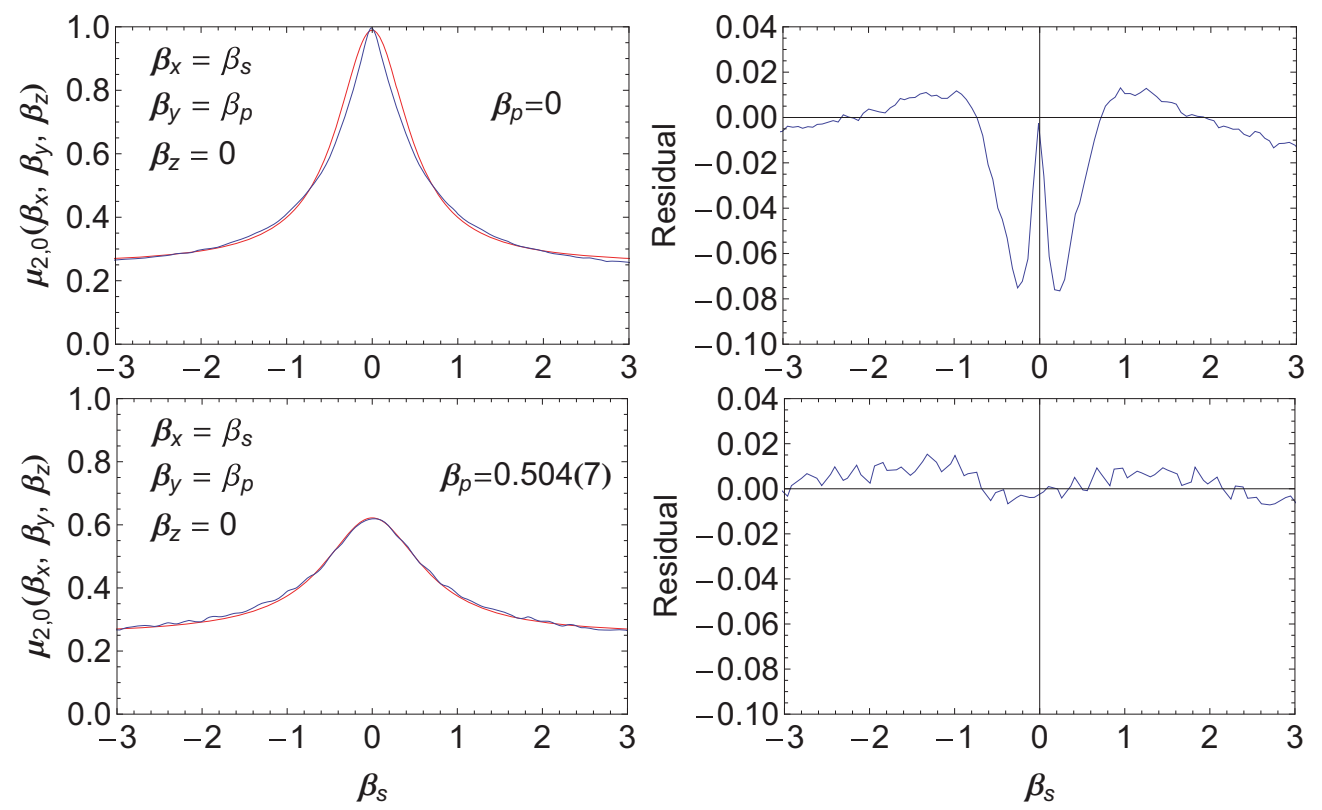

FIG. 10. (Color online) (Left) Experimental line shapes of the transverse GSHE for different values of $\beta_{p}=\beta_{\perp}$ and fit with Eq. (22). (Right) The amplitudes of the residual.

The essential physical mechanisms discussed therein also apply to the present study. In the uncoated vacuum cell used in the experiments described here, a single wall collision of a polarized atom randomizes its spin orientation. As a consequence, the resonance line shape slightly differs from an absorptive Lorentzian, as discussed in Ref. [25]. This effect is small as evidenced by the results shown in Fig. 10. The upper graphs show a transverse Hanle resonance in absence of residual fields, together with a fit by the Lorentzian line shape model function (10) and the fit residuals, which vary by a few percent of the total signal amplitude. The lower graphs show the same resonance in presence of a small $\left(\beta_{\mathrm{p}} \approx 0.5\right)$ transverse magnetic field, in which case the residuals are smaller. The conclusion is that the Lorentzian fits yield signal amplitudes with a systematic uncertainty on the order of few percent.
[1] D. Budker and M. Romalis, Nat. Phys. 3, 227 (2007).

[2] S. Knappe, in MEMS Atomic Clocks (Elsevier, Amsterdam, 2009), pp. 572-605.

[3] P. Marte, P. Zoller, and J. L. Hall, Phys. Rev. A 44, 4118 (1991).

[4] L. V. Hau, S. E. Harris, Z. Dutton, and C. H. Behroozi, Nature (London) 397, 594 (1999).

[5] C. Liu, Z. Dutton, C. H. Behroozi, and L. V. Hau, Nature (London) 409, 490 (2001).

[6] D. Budker, W. Gawlik, D. F. Kimball, S. M. Rochester, V. V. Yashchuk, and A. Weis, Rev. Mod. Phys. 74, 1153 (2002).

[7] W. Hanle, Z. Phys. 30, 93 (1924).

[8] J. C. Lehmann and C. Cohen-Tannoudji, C. R. Acad. Sci. Paris 258, 4463 (1964).

[9] N. Castagna and A. Weis, Phys. Rev. A 84, 053421 (2011).

[10] F. Renzoni, W. Maichen, L. Windholz, and E. Arimondo, Phys. Rev. A 55, 3710 (1997).

[11] C. Andreeva, S. Cartaleva, Y. Dancheva, V. Biancalana, A. Burchianti, C. Marinelli, E. Mariotti, L. Moi, and K. Nasyrov, Phys. Rev. A 66, 012502 (2002).

[12] A. Sargsyan, D. Sarkisyan, and A. Papoyan, Phys. Rev. A 73, 033803 (2006).
[13] E. Arimondo, Progr. Opt. Tech. Rep. 35, 259 (1996).

[14] A. V. Taichenachev, A. M. Tumaikin, and V. I. Yudin, Phys. Rev. A 61, 011802 (1999).

[15] J. Dimitrijević, A. Krmpot, M. Mijailović, D. Arsenović, B. Panić, Z. Grujić, and B. M. Jelenković, Phys. Rev. A 77, 013814 (2008).

[16] M. Auzinsh, R. Ferber, F. Gahbauer, A. Jarmola, and L. Kalvans, Phys. Rev. A 78, 013417 (2008).

[17] A. Vilardi, D. Tabarelli, L. Botti, A. Bertoldi, and L. Ricci, J. Phys. B 42, 055003 (2009).

[18] S. I. Kanorsky, A. Weis, J. Wurster, and T. W. Hansch, Phys. Rev. A 47, 1220 (1993).

[19] A. Weis, J. Wurster, and S. I. Kanorsky, J. Opt. Soc. B 10, 716 (1993).

[20] A. Weis, G. Bison, and A. S. Pazgalev, Phys. Rev. A 74, 033401 (2006).

[21] W. Happer, Rev. Mod. Phys. 44, 169 (1972).

[22] K. Blum, Density Matrix Theory and Applications, 2nd ed. (Plenum Press, New York, 1996).

[23] M. Auzinsh, D. Budker, and S. M. Rochester Optically Polarized Atoms (Oxford University Press, New York, 2010). 
[24] C. Cohen-Tannoudji and J. Dupont-Roc, Opt. Commun. 1, 184 (1969).

[25] E. Pfleghaar, J. Wurster, S. I. Kanorsky, and A. Weis, Opt. Commun. 99, 303 (1993).

[26] A. P. Kazantsev, V. S. Smirnov, A. M. Tumaikin, and I. A. Yagofarov, Opt. Spectrosc. (USSR) 57, 116 (1984).

[27] A. M. Akulshin, S. Barreiro, and A. Lezama, Phys. Rev. A 57, 2996 (1998).
[28] A. Lezama, S. Barreiro, and A. M. Akulshin, Phys. Rev. A 59, 4732 (1999).

[29] Y. Dancheva, G. Alzetta, S. Cartaleva, M. Taslakov, and C. Andreeva, Opt. Commun. 178, 103 (2000).

[30] J. Alnis and M. Auzinsh, J. Phys. B 34, 3889 (2001).

[31] E. Breschi, G. Kazakov, R. Lammegger, G. Mileti, B. Matisov, and L. Windholz, Phys. Rev. A 79, 063837 (2009).

[32] Y. Xiao, Mod. Phys. Lett. B 23, 661 (2009). 